\title{
Social Structure, Endogenous Diversity, and Collective Accuracy
}

\author{
Evan Economo, Lu Hong, and Scott E Page*
}

January 6, 2016

\begin{abstract}
Markets, democracies, and organizations rely on accurate aggregate predictions to function properly. A large literature explains how accuracy can arise from diverse predictive models, typically captured as independent or non perfectly correlated signals. Yet, that literature largely ignores how the diversity of models arises and is maintained. In this paper, we derive equilibrium levels of model diversity as a function of social structure, population size, the probability of experimentation, and the number of available models by building on a theoretical framework used to study biodiversity. We then link model diversity to collective accuracy by generalizing the bias-variance decomposition formula. Assuming equally accurate models, we find that for large populations collective accuracy depends primarily on the diversity of available models and that for small populations, social structure and rates of experimentation also matter. We then show, contrary to intuition, that dividing a population into isolated sub groups does little to increase equilibrium diversity levels. We also extend the model to allow for heterogeneity in accuracy and selection effects.
\end{abstract}

Keywords: Diversity, Collective Wisdom, Social Structure, Conformity

JEL Classification: $D 70, D 83$

\footnotetext{
${ }^{*}$ Scott E Page, University of Michigan and Santa Fe Institute Scott acknowledges the NSF, ARO, and IARPA for funding on this project. Lu Hong, Department of Finance, Loyola University. Evan Economo Okinawa Institute of Science and Technology.
} 


\section{Introduction}

Democracies, markets, and organizations require collections of individuals to make accurate aggregate forecasts of future events and values in order to function properly (Ober 2008, Von Hayek 1945). These forecasts or predictions need not be exact, but they do need to lie within reasonable bounds. When predictions miss the mark by wide margins, costly consequences ensue. Capital is misallocated, firms fail, markets crash (Reinhart and Rogoff 2009), and the masses can even fall prey to illogical ideas (Whyte 1989, Le Bon 1895).

The logic that underlies the possibility of collective accuracy is formalized in the Condorcet Jury Theorem. If individuals receive independent signals, their errors will cancel out. ${ }^{1}$ More elaborate models extend that core insight and demonstrate that collective accuracy depends on (i) the difficulty of the predictive task, (ii) the level of uncertainty, (iii) the extent of social influence, (iv) the amount of model diversity, (v) the rule or mechanism used to aggregate predictions (Armstrong 2001, Piketty 1999, Lamberson and Page 2012) (vi) whether or not forecasts are weighted, ${ }^{2}$ and (vii) whether individuals behave strategically (Feddersen and Pesendorfer 1997).

The extant literature on collective accuracy, though vast, takes the distribution of model and signal accuracy to be an exogenous feature of the world (Page 2014). ${ }^{3}$ In this paper, we demonstrate that it is possible to endogenize the distribution of signals. Specifically, we derive the equilibrium level of predictive model diversity as a function of social structure, rates of experimentation, the number and accuracy of possible models, and the extent of selection. We then show that this equilibrium diversity level is a sufficient statistic for collective accuracy by generalizing the familiar bias-variance decomposition formula.

In our model, we assume a population of individuals embedded in a social structure. Each individual relies on a model to make a prediction about some future event. Individuals can copy models from others or draw new models from a set of possible models. The accuracy of the population at any moment will depend on the diversity of models in use. We intend for this framework to approximate many real world situations. For example, within a group of doctors evaluating a patient, there will exist multiple predictive models for the cause of some symptom, and among economic forecasters, there will

\footnotetext{
${ }^{1}$ See Groffman, Owen, and Feld (1983) for a survey.

${ }^{2}$ see Armstrong 2001, Ashton 1986, Batchelor and Dua 1995, Lamberson and Page 2012, Breiman 1996, Welinder et al 2010 .

${ }^{3}$ Variation in the realizations of signal values are typically explained by environmental variation, noise, or complexity of the inferential task (Page 2007).
} 
exist multiple predictive models for the future path of the economy. The same is true in other domains such as fashion, politics, sports, and the arts. In each case, there will exist a set of plausible models distributed across the relevant population in some way.

To solve for equilibrium diversity, we apply and extend theoretical frameworks developed for the study of diversity dynamics in biological systems (Kimura and Crow 1964, Hartl \& Clark 1997, Hubbell 2001). Those frameworks capture the forces that shape the diversity and distributions of biological variants, be it the diversity of genotypes in a population or species in an ecosystem. Specific models show how diversity levels are shaped through introduction of novelty (i.e. through mutation/speciation), the effects of sampling error in a finite system that dissipate diversity from a system (i.e. drift), and differences in fitness among variants (i.e. selection). Here, we take a similar approach to study the forces shaping the diversity of predictive models in a social group.

Reinterpreting biological models with social contexts is not novel. Evolutionary game theory has been applied to the dynamics of competitive types in both biology and economics for decades (Maynard Smith 1982, Friedman 1998). To date though, most models have emphasized selection. A large class of biological models, called neutral models, do not assume selection. These models are used to explore the diversity levels that arise and are maintained when dynamics are governed by mutation and drift (Kimura 1984, Hubbell 2001). ${ }^{4}$

Neutrality also applies to socio-economic systems. It does so in contexts when there exist multiple signals or models of approximately equal accuracy or when the ability to discern model accuracy is insufficient to choose among models. In both cases, there exists a set of equally plausible models circulating in the population, and the social learning process exhibit neutral drift where changes in population distribution result from sampling errors and stochastic fluctuations. Selection plays no role.

Neutral drift does not imply that all models are equally likely to spread in a population. Social structure can advantage some models. Empirical analyses of how people think about the world and what models they use find that individuals often borrow the models and ideas of their friends. ${ }^{5}$ It follows that in hierarchal societies, the models adopted by high influence individuals proliferate with higher probability. In other words, the source may be as important as the content.

To account for the fact that in many social contexts selection does occur, we later extend the model

\footnotetext{
${ }^{4}$ We make no claims to the originality of the idea that social and biological processes may exhibit similar dynamics. See for example Cavalli-Sforza and Feldman (1981).

${ }^{5}$ See, for example, Huckfeldt, Johnson, and Sprague (2004) for an analysis of how voters change their ideas and opinions.
} 
to allow for different accuracy levels as well as selection. We find that selection is a double edged sword. It selects better models but reduces diversity. The former effect improves accuracy while the latter reduces it.

In the initial model, we assume an exogenous set of available predictive models. Initially, individuals randomly draw a predictive model from this set. Over time the distribution across the models evolves according to a social process that includes experimentation, in which an individual randomly draws a new model from the set of possible models, and conformity, in which an individual copies the predictive model of someone else in the population. Varying the rates of experimentation and conformity alters the equilibrium level of diversity. As would be expected, more experimentation produces more diversity and, as a result, greater collective accuracy.

The rate of experimentation can be interpreted as how often individuals form their own opinions, and the rate of conformity can be interpreted as capturing social influences, with experimentation introducing diversity into the population and conformity purging it. Though here we consider predictive models as our unit of analysis, the same formalism could be applied to the study of cultural traits or behaviors (Bentley, Hahn, and Shennan 2004, Bednar et al 2010, Page 2014). The mechanism through which people copy can also be made more elaborate. Individuals could, for example, refer back several periods rather than copy a current model (Omerod 2012, Bentley, Ormerod, and Batty 2011). ${ }^{6}$

Our formal assumptions can be motivated by the following structural model: Each type of predictive model can be based on a set of attributes which in turn determine the value being forecasted. ${ }^{7}$ Different models rely on different attributes. We can then assume that each model includes the same number of attributes and that each attribute is equally informative and independent of the other attributes. Together, these assumptions imply that each type of predictive model has identical accuracy. They also imply that the correlation between two predictive models depends on the overlap between the variables they contain: the greater the overlap, the higher the correlation.

We solve for equilibrium diversity levels by using a master equation. That equation calculates the equilibrium probability that two individuals use the same type of model next period as a function of that probability in the previous period. One methodological contribution of this paper is to show that

\footnotetext{
${ }^{6}$ Our approach differs from papers that study the spread of innovation (Young 2009). By definition, innovations improve outcomes (provided there exist enough adopters). That's not to say that we don't consider similar questions. For example, Young (2011) shows how social structure in the form of tighter clustering allows innovations to spread more quickly through a population.

${ }^{7}$ Seen as statistical signals, these predictive models can therefore be classified as interpreted and not generated signals (Hong and Page 2009).
} 
we can solve this master equation for a variety of social structures: random mixing, non interacting subpopulations, and a hierarchy, where the population is divided into an elite upper group and a lower group. In this last case, people only copy the models of the higher social status upper group.

The second methodological contribution is to prove that the diversity index is a sufficient statistic for the collective accuracy of the population. In other words, one need not know the entire distribution over models to calculate the expected collective accuracy. One need only know the diversity index. This is the aforementioned extension of the bias-variance decomposition theorem.

Our substantive contributions demonstrate how for each social structure the equilibrium diversity (and therefore the collective accuracy) depends on measurable features of the social world such as population size, the diversity of possible models, rates of experimentation, and rates of conformity. We show, not unexpectedly, that hierarchical social structures reduce diversity and that adding more available predictive models increases diversity as does increasing the population size. Even though one can intuitively discern the direction of these effects, one cannot necessarily assess their relative magnitudes or the effect sizes. We show, for example, that for large groups, the social structure, e,g, whether it is hierarchical or divided into non interacting subgroups, has almost no effect. In contrast, the number of possible models proves to be a very important parameter.

Among our more surprising results is that breaking the population into non interacting subgroups, which would seem to increase diversity, has little to no effect except in the case of very small populations. Though each subpopulation differs markedly from the others, each subpopulation maintains much less diversity than can be maintained by the entire population. This lack of diversity within the subgroups offsets - almost exactly - the gain in diversity from the differences between the groups, producing a negligible net effect on diversity.

Our social process model owes a substantial intellectual debt to models linking diversity with population/community structure used in ecology and population genetics (Kimura and Crow 1964, Whitlock \& Barton 1997, Economo \& Keitt 2008). The primary differences between our model and those models are two fold. First, we interpret and use our model differently because of differences between distributions of species and distributions of predictive models. It follows that the relevant ranges of parameters used in the model differ markedly from those used by ecologists. For example, rates of experimentation in humans may be higher than genetic mutation rates and while we will consider groups of fewer than one hundred people, populations of viruses tend to be orders of magnitudes larger. Thus, even though in 
our random mixing case, we write nearly identical equations, we explore distinct parts of their domains. Second, as mentioned, our model departs from the literature in ecology and population genetics in that we consider social structures, such as hierarchies, that capture interactions among groups of people.

The remainder of the paper consists of five parts. We first describe a framework for relating social structure and process to equilibrium diversity levels. We then generalize the bias-variance decomposition theorem to connect diversity to collective accuracy. By combining these results with the first part, we derive results relating social structure and process to collective accuracy. In the fourth part, we use numerical simulations to corroborate the theoretical findings and to clarify the relative magnitudes of the effects of changing social structure, the diversity of models, and the rates of experimentation and conformity, for large, moderate, small populations. In the fifth part, we introduce the possibility of selection effects, and in the final part, we describe other applications of our model and discuss possible extensions.

\section{Social Structure and Population Diversity}

We assume a population of size $N$ in which each individual takes on one of $K$ finite types that are assigned independently by an external process. ${ }^{8}$ Later in the paper, we interpret these types as predictive models. In a more general model of social dynamics, these types could represent anything from consumer choices in a category, to political party affiliations, to styles of dress.

We denote the distribution produced by this external process, $G$, as a multinomial distribution over the $K$ types: $\left(q_{1}, q_{2}, \ldots, q_{K}\right)$. For both this distribution and for the equilibrium distribution, we rely on the diversity index as a measure of heterogeneity. This index equals the inverse of the probability that two draws from the distribution have the same type $f(G),{ }^{9}$

$$
D(G)=\frac{1}{f(G)}=\frac{1}{\sum_{j=1}^{K}\left(q_{j}\right)^{2}}
$$

Notice that if each of the $K$ types is equally likely then $f(G)=\frac{1}{K}$ and $D(G)=K$. For this reason, the diversity index is often called the effective number of types. ${ }^{10}$

\footnotetext{
${ }^{8}$ The model shares a resemblance to the Wright-Fisher infinite alleles model of population genetics (Kimura \& Crow 1964). One difference is that population geneticists can ignore second order effects of the rates of experimentation because they consider low mutation rate. The more important difference is that we embed the model within social structures.

${ }^{9}$ In ecology, this is known as Simpson's Index. In economics it's called the Herfindahl-Hirschman Index.

${ }^{10}$ See Page (2010) for a list of diversity measures.
} 
Individuals change their type through two processes: experimentation and conformity. Experimentation entails randomly selecting a new type according to the distribution $G$. Conformity entails copying the type of some other member of the population. We capture these effects using a discrete time representation of the dynamics. Time is divided into discrete intervals of arbitrarily small length. During each interval all individuals update synchronously. ${ }^{11}$

In each time interval, an individual takes one of two actions. With probability $\nu$ an individual experiments by drawing a new type using the external process $G$, and with probability $(1-\nu)$, an individual randomly copies the type of another individual. The individual to be copied will depend on the social structure in a way we make formal below. ${ }^{12}$

\section{Random Mixing}

We first consider random mixing in which individuals randomly choose someone to copy. To describe the dynamics, we introduce the concept of a progenitor. For a given individual, we define its progenitor recursively as follows: Begin at the present instant, look to see when the individual last either copied another individual or experimented from the external process. If the individual last experimented, then it is its own progenitor. If it copied another individual, then its progenitor is the progenitor of that individual.

We define the progenitor of the other individual using the same recursive rule. Given this construction, progenitors must be identified by an individual and a time period. Formally, we let $A(i, t)$ denote individual i's progenitor at time $t$. If $A(i, t)=(j, \tau)$, this means that time $t$ individual $i$ 's progenitor is time $\tau$ individual $j$, i.e., at $t$, individual $i$ is a copy of individual $j$ at time $\tau$. Figure ?? illustrates this concept.

As a crucial middle step in deriving the equilibrium level of diversity in the population, we use the concept of progenitor to first derive, $a$, the probability that two individuals sampled from the population without replacement have the same progenitor. We do so by writing a difference equation for $a$ given random mixing in terms of the probability of experimentation $\nu$, the number of models $K$, and the population size $N$. Let $a^{\prime}$ denote the value of $a$ in the next time period. We then obtain the following:

\footnotetext{
${ }^{11}$ These assumptions greatly reduce the notation. Continuous time dynamics produce qualitatively similar results.

${ }^{12}$ The dynamics assume that at each moment an individual either experiments or copies someone in the population. As will be clear when we describe the dynamics formally, were we to assume that the experimentation probability plus the conformity probability sum to less than one, then the equilibrium would not change. This could be done by assuming that with probability $(1-r)$ the individual takes no action and that experimentation and copying occur with probabilities $r \nu$ and $r(1-\nu)$. The only effect of such a change would be to slow the dynamics.
} 


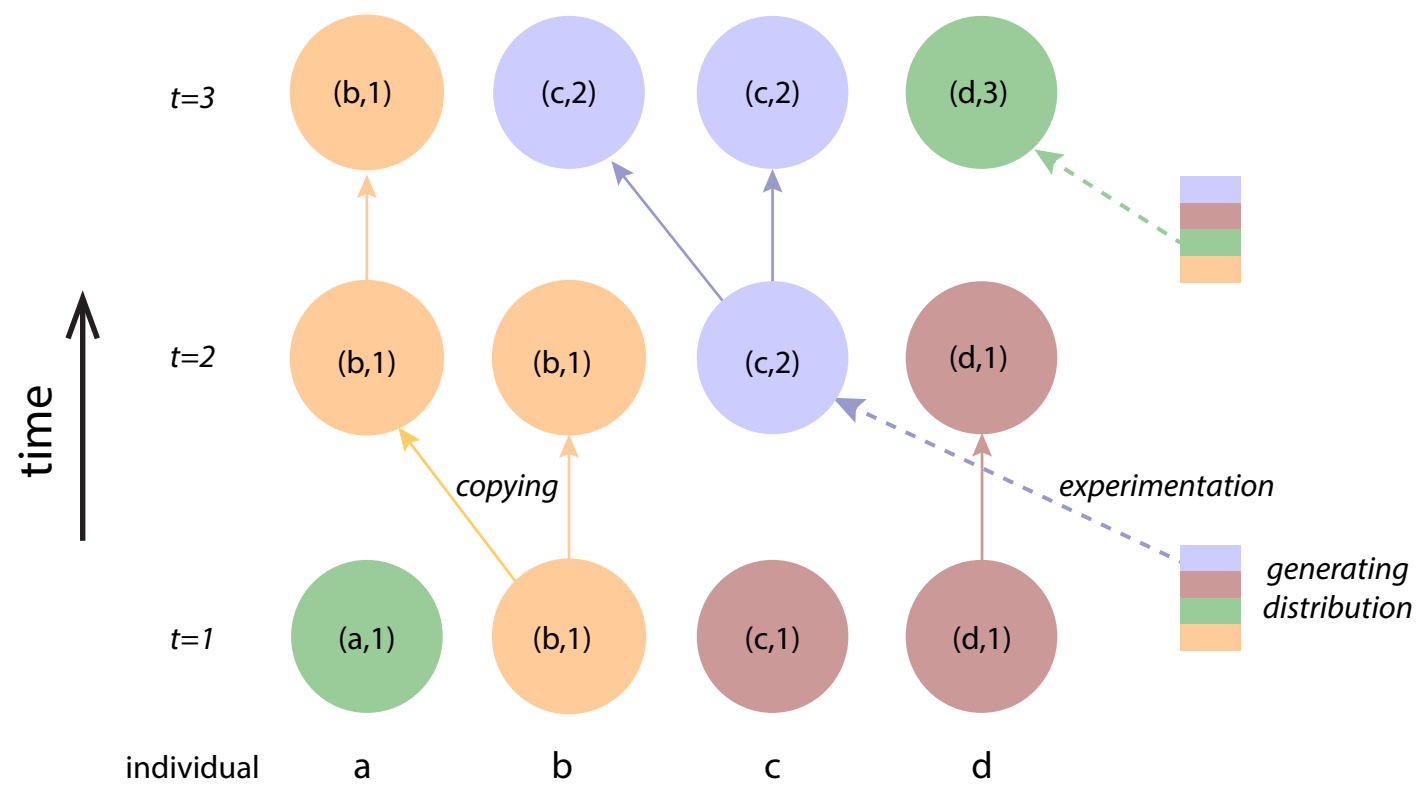

Figure 1: An example population with four individuals (a-d). Individuals update their "model" either by copying another individual or experimenting (i.e. choosing a model from the generating distribution). At each point in time, an individual can trace her opinion to a progenitor, the individual/time-point where that model entered the population.

Figure 1: An example population with four individuals (a-d). Individuals update their model either by copying another individual or experimenting (i.e. choosing a model from the generating distribution). At each point in time, an individual can trace her opinion to a progenitor, the individual/time-point where that model entered the population.

$$
\left(a^{\prime}-a\right)=-a\left\{\nu^{2}+2 \nu(1-\nu)+(1-\nu)^{2}\left[\frac{N-1}{N}(1-a)\right]\right\}+(1-a)(1-\nu)^{2}\left(\frac{1}{N}+\frac{N-1}{N} a\right)
$$

This expression can be explained as follows: The first term equals the probability that two individuals who began with the same progenitor now have different progenitors. This equals the sum of the probabilities that both experiment (prob. $\nu^{2}$ ), that one experiments and the other copies (prob. $2 \nu(1-\nu)$ ), and that both copy (prob. $\left.(1-\nu)^{2}\right)$ but they happen to copy individuals with different progenitors. This last term is computed by multiplying the probability that they copy different individuals, $\frac{N-1}{N}$, and the probability that those individuals have different progenitors, $(1-a)$. 
The second term equals the probability that two individuals who began with different progenitors acquire the same progenitor. This occurs when two individuals either copy the same individual (prob. $\frac{1}{N}$ ) or copy different individuals who have the same progenitor (prob. $\frac{N-1}{N} a$ ). Simplifying the right side of the equation gives

$$
\left(a^{\prime}-a\right)=-a+(1-\nu)^{2}\left(\frac{1}{N}+\frac{N-1}{N} a\right)
$$

We define a stochastic equilibrium to exist when the expected probability that two individuals have the same progenitor does not change, i.e. when $a^{\prime}$ equals $a$. Setting $\left(a^{\prime}-a\right)$ equal to 0 in the previous equation, we obtain the equilibrium value $a^{*}$

$$
a^{*}=\frac{(1-\nu)^{2}}{N\left(2 \nu-\nu^{2}\right)+(1-\nu)^{2}}
$$

To solve for collective accuracy, we will need to know the probability that two individuals have the same progenitor, to compute the probability that they have the same type. To do that, we need to introduce the following notation: Let $\Delta$ denote the diversity index of types in the population in equilibrium. Then, by definition, $\frac{1}{\Delta}$ represents the probability that two randomly drawn individuals from the population with replacement have the same type in equilibrium. We have the following claim.

Claim 1 For a randomly mixing population, the equilibrium probability that two randomly chosen (with replacement) individuals have the same type equals

$$
\frac{1}{\Delta}=1-\left[1-\frac{1}{N\left(2 \nu-\nu^{2}\right)+(1-\nu)^{2}}\right](1-f(G))
$$

pf. Note first that $\frac{1}{\Delta}$ must take into account the possibility that two distinct progenitors have the same type. We first solve for the probability that two individuals drawn with replacement do not have the same type. This equals the joint probability of three independent events: two different individuals are drawn, they have distinct progenitors, and the distinct progenitors have distinct types.

$$
1-\frac{1}{\Delta}=\left(1-\frac{1}{N}\right)(1-a)(1-f(G))
$$


We substitute in the equilibrium value for $a$. This gives the following expression:

$$
1-\frac{1}{\Delta}=\left(1-\frac{1}{N}\right)\left(1-\frac{(1-\nu)^{2}}{N\left(2 \nu-\nu^{2}\right)+(1-\nu)^{2}}\right)(1-f(G))
$$

Rearranging terms and solving for $\frac{1}{\Delta}$ gives the result.

\section{The Dynamics of the Process}

Keep in mind that this is a stochastic equilibrium. A finite population will tend to this average value but the time series for the diversity index will exhibit substantial variation. We can use our simplified expression for the change in the probability of a common progenitor, $\left(a^{\prime}-a\right)$, to investigate the dynamics of the process. The dynamics are quite straightforward and produce a unique equilibrium that is both locally and globally stable.

Assume that initially, the probability of having a common progenitor equals zero, i.e. $a=0$. Using the expression for $\left(a^{\prime}-a\right)$, the probability of matching increases by the probability that two individuals copy the same individual $\left((1-\nu)\right.$ squared times $\left.\frac{1}{N}\right)$. As $a$ increases, the rate of increase in the probability of having the same progenitor decreases. ${ }^{13}$ So the process will quickly head toward equilibrium and then slow near the equilibrium. Alternatively, suppose that we begin the process by assigning the same progenitor to all agents. The probability of having the same progenitor will fall quickly but then slow near the equilibrium.

Figure ?? which shows sample paths from numerical simulations using one hundred agents and ten models. In the figure, the runs vary based on the rates of experimentation. The graph on the top shows the population dynamics with a high rate of experimentation $(\nu=0.5)$. The middle and lower graphs show moderate $(\nu=0.05)$ and low rates of experimentation $(\nu=0.005) .{ }^{14}$

Notice first the aforementioned fluctuations. Even though we can solve for expected equilibrium diversity levels, those equilibria are highly stochastic. Actual levels fluctuate dramatically. Note next how diversity falls as the probability of experimentation decreases because experimentation increases diversity by introducing new types. Lowering experimentation implies more conformity so that fewer types are maintained in the population. This can be seen in the line graphs below the colored distribution

\footnotetext{
${ }^{13}$ The change in the value has two components: an increase of $(1-\nu)^{2} \frac{1}{N}$ and a decreases of $a\left(1-(1-\nu)^{2} \frac{(N-1)}{N}\right)$. The second term is larger.

${ }^{14}$ Each of the runs was initiated with a single type so that the graphs would not be distorted by the initial conditions.
} 
graphs. Average diversity is much higher in the top graph (high experimentation) than in the other two cases.

Finally, note that with high levels of experimentation (the top graph), diversity levels fluctuate rapidly but within a tight interval. That's not true of the two cases with lower rates of experimentation. In those cases, diversity levels fluctuate substantially over time. With low experimentation there exist long periods in which one or two models can dominate the population.

\section{Isolated Groups}

We next change the social structure and split the population $N$ into $H$ non-interacting groups each of size $\frac{N}{H}$, capturing the idea that an individual can only copy someone from the same group. We refer to these as isolated groups. We again solve for the probability that two randomly chosen individuals have the same type.

Claim 2 When the population is divided into $H$ equally sized, isolated groups, the equilibrium probability that two randomly chosen (with replacement) individuals have the same type is given by the following expression:

$$
\frac{1}{\Delta_{I}}=1-\left(1-\frac{1}{N\left(2 \nu-\nu^{2}\right)+H(1-\nu)^{2}}\right)(1-f(G))
$$

pf. As in the previous claim, we first solve for the probability that the two individuals do not have the same type. This equals:

$$
1-\frac{1}{\Delta_{I}}=\left(1-\frac{1}{H}\right)(1-f(G))+\frac{1}{H}\left(1-\frac{H}{N}\right)\left(1-a_{h}\right)(1-f(G))
$$

The first term equals the probability that the two individuals come from different clusters times the probability that two independent samples from the generator are of different types. The second term equals the probability of sampling from the same cluster times the probability of sampling two distinct individuals times the probability these two individuals have different progenitors, times the probability those progenitors have different types. 
From our results that assumed random mixing and adjusting for population size within a group, $\frac{N}{H}$, it follows that

$$
\left(1-\frac{H}{N}\right)\left(1-a_{h}\right)=1-\frac{1}{\frac{N}{H}\left(2 \nu-\nu^{2}\right)+(1-\nu)^{2}}
$$

Rearranging terms completes the proof.

We now state a claim relating the diversity of a randomly mixed population to a population of isolated groups.

Claim 3 Splitting the population into non interacting groups decreases the expected probability that two individuals will have the same type.

pf. From the previous two claims:

$$
\frac{1}{\Delta_{I}}=\frac{1}{\Delta}-\left(\frac{1}{N\left(2 \nu-\nu^{2}\right)+(1-\nu)^{2}}-\frac{1}{N\left(2 \nu-\nu^{2}\right)+H(1-\nu)^{2}}\right)(1-f(G))
$$

Since $H>1, \frac{1}{\Delta_{I}}<\frac{1}{\Delta}$.

Note that when $H=1$ we get the same expression as in the random case. As a population is divided into more groups, the probability that two individuals have the same type falls like $\frac{1}{H}$. Note that this fall is not as precipitous as one might expect because each smaller group can maintain less diversity.

\section{Hierarchical Groups}

Last, we consider a structure where the population is divided into two groups arranged in a hierarchy: an elite upper group with $U$ members and a lower group with $L=(N-U)$ members. ${ }^{15}$ In this social structure, individuals in both groups only copy members of the upper group. Given these assumptions, the dynamics for the upper group can be considered as a group of size $U$ with random mixing. The

\footnotetext{
${ }^{15}$ All of the models are equally accurate. The elites, or the upper group, are no better at making predictions than the lower group. This assumption makes sense provided that belonging to the upper group depends on criteria that are independent of model accuracy such as charisma, confidence, or tenure. The assumption would be problematic if the individuals in the upper group have achieved their status based on the performance of their models. One can easily write an extension of the model to allow for the elites to be more accurate. Doing so would not change the equilibrium diversity levels but it would affect population accuracy because the models that exist in equilibrium disproportionately belong to the upper group.
} 
expected change in the probability that two individuals in the upper group have the same progenitor can be written as:

$\left(a_{U U}^{\prime}-a_{U U}\right)=-a_{U U}\left[\nu^{2}+2 \nu(1-\nu)+(1-\nu)^{2}\left[\frac{U-1}{U}\left(1-a_{U U}\right)\right]\right)+\left(1-a_{U U}\right)(1-\nu)^{2}\left(\frac{1}{U}+\frac{U-1}{U} a_{U U}\right)$

In equilibrium, two individuals in the upper group will have the same progenitor with probability

$$
a_{U U}^{*}=\frac{(1-\nu)^{2}}{U\left(2 \nu-\nu^{2}\right)+(1-\nu)^{2}}
$$

To compute the equilibrium probability that an individual in the lower group and an individual in the upper group have the same progenitor, we write the difference equation for $a_{U L}$, the probability that they have the same progenitor, as follows:

$\left(a_{U L}^{\prime}-a_{U L}\right)=-a_{U L}\left(\nu^{2}+2 \nu(1-\nu)+(1-\nu)^{2}\left[\frac{U-1}{U}\left(1-a_{U U}\right)\right]\right)+\left(1-a_{U L}\right)(1-\nu)^{2}\left(\frac{1}{U}+\frac{U-1}{U} a_{U U}\right)$

From this expression, it follows that $a_{U L}=a_{U U}$. Logically, this follows because individuals in the two groups both only copy individuals in the upper group. Similarly, we can write the difference equation for $a_{L L}$ as follows:

$\left(a_{L L}^{\prime}-a_{L L}\right)=-a_{L L}\left(\nu^{2}+2 \nu(1-\nu)+(1-\nu)^{2}\left[\frac{U-1}{U}\left(1-a_{U U}\right)\right]\right)+\left(1-a_{L L}\right)(1-\nu)^{2}\left(\frac{1}{U}+\frac{U-1}{U} a_{U U}\right)$

It follows also that $a_{L L}$ also equals $a_{U U}$. We can therefore state the following claim:

Claim 4 When the population is divided into an upper and lower group, and individuals only copy the types of individuals in the upper group, then the equilibrium probability that two randomly chosen (with replacement) individuals have the same type is given by the following expression:

$$
\frac{1}{\Delta_{H}}=1-\left(1-\frac{1}{N}\right)\left(1-\frac{(1-\nu)^{2}}{U\left(2 \nu-\nu^{2}\right)+(1-\nu)^{2}}\right)(1-f(G))
$$

pf. As in the previous claims solving for equilibrium diversity, we first solve for the probability that two individuals do not have the same type. This equals the following: 


$$
1-\frac{1}{\Delta_{H}}=\left(1-\frac{1}{N}\right)\left(1-\frac{(1-\nu)^{2}}{U\left(2 \nu-\nu^{2}\right)+(1-\nu)^{2}}\right)(1-f(G))
$$

Rearranging terms and solving for $\frac{1}{\Delta_{H}}$ gives the result.

In light of the previous claim, we can state a claim that compares the equilibrium diversity for our hierarchical social structure with that of random mixing.

Claim 5 Creating a hierarchy where individuals in the lower group copy the types of individuals in the upper group increases the probability that two individuals will have the same type relative to random mixing.

pf. Equations (??) and (??) state that

$$
\frac{1}{\Delta_{H}}=1-\left(1-\frac{1}{N}\right)\left(1-\frac{(1-\nu)^{2}}{U\left(2 \nu-\nu^{2}\right)+(1-\nu)^{2}}\right)(1-f(G))
$$

and

$$
\frac{1}{\Delta}=1-\left(1-\frac{1}{N}\right)\left(1-\frac{(1-\nu)^{2}}{N\left(2 \nu-\nu^{2}\right)+(1-\nu)^{2}}\right)(1-f(G))
$$

The result follows from the fact that $U<N$.

These claims establish that diversity increases by creating isolated populations and decreases by creating a hierarchy. More importantly though, they give closed form solutions for the magnitude of those changes, a fact that we exploit later in the paper.

\section{Collective Accuracy}

Having solved for equilibrium levels of diversity as a function of the social structure (random mixing, isolated groups, and hierarchy) and process (the relative probability of experimentation, the population size, and the diversity of available models), we now derive an explicit relationship between the diversity of predictive model types and collective accuracy. 


\section{Predictive Models}

In what follows, we assume a discrete set of periods. Prior to each period, a multi-dimensional state of the world is revealed, and the individuals apply their predictive models. We will refer to the dimensions in the state of the world as attributes.

To provide some interpretations of this construction, think of the attribute values as corresponding to say current market conditions, the features of a policy proposal in a democracy, or the actions of a competing business. One can therefore think of each period's state as a "case." Individuals predict a sequence of cases.

Formally, we assume that the state of the world can be represented by a real valued vector of length $M$ where each component of the vector is a random variable with mean zero and standard deviation one. These components' values are independent of one another.

The state of the world (at time $t$ ), $x \in R^{M}$, is a vector $x_{j} \sim N(0,1)$ for $j=1$ to $M$ and all $x_{j}$ 's are independent.

We then assume a functional relationship between states of the world and outcomes, enabling us to link attributes to predictive model accuracy and correlation. For the purposes of this paper, we assume a value function $\chi$ that depends linearly on the attributes denoted by $x_{j}$.

$$
\chi(x)=\sum_{j=1}^{M} x_{j}
$$

The framework can be extended to allow for nonlinear effects provided that the value function is symmetric with respect to each attribute. Each individual $i$ considers some subset of attributes $M_{i} \subset M .{ }^{16}$ Hereafter, we refer to each subset as a model type. The prediction, $\chi_{i}$, of individual $i$ equals the sum of it's attribute values:

$$
\chi_{i}(x)=\sum_{j \in M_{i}} x_{j}
$$

\footnotetext{
${ }^{16}$ For convenience, we let $M$ denote both the set of attributes and the cardinality of that set.
} 
The collective prediction in state $x$ equals the average of the individual predictions:

$$
\frac{1}{N} \sum_{i=1}^{N} \chi_{i}(x)
$$

In what follows, we assume that each individual considers exactly $R$ attributes so that each individual is equally good at predicting outcomes. ${ }^{17}$ As an example, consider a venture capital firm that evaluates start up companies. The relevant attributes of a start up might include the capabilities of the entrepreneur, her experience, the number of competitors, the expected time to market, the feasibility of the technology, and the potential markets and spin-offs. Any one venture capitalist within this firm might consider some subset of those attributes. Another may consider a different subset of attributes. Yet, each may be equally good at predicting outcomes. Collectively, the firm will predict more accurately if they maintain a diversity of models.

Of course, collective accuracy depends on more than just the diversity of models. It also depends on their individual accuracy. The expected error of a predictive model depends on $V=M-R$, the number of attributes not included by an individual in her model. Given our construction, the variance of forecast error, $\sigma_{i}^{2}$, equals $V$ for all $i$. Also, the covariance between the errors of any two predictions will depend on the overlap in the attributes that they do not consider. That covariance can be found using the following formula:

$$
\begin{aligned}
E\left[e_{i}(x) \cdot e_{i^{\prime}}(x)\right] & =E\left[\left(\sum_{j \notin M_{i}} x_{j}\right) \cdot\left(\sum_{j \notin M_{i^{\prime}}} x_{j}\right)\right] \\
& =E\left[\sum_{j \notin M_{i} \cup M_{i^{\prime}}}\left(x_{j}\right)^{2}\right]
\end{aligned}
$$

Here, $e_{i}(x)=\chi_{i}(x)-\chi(x)$, and $e_{i^{\prime}}(x)$ is defined accordingly. Let $C_{i i^{\prime}}=\left|M \backslash\left\{M_{i} \cup M_{i^{\prime}}\right\}\right|$. It follows then that the covariance of any two randomly selected distinct predictive models equals $C_{i i^{\prime}}$. Note that by construction $C_{i i^{\prime}}<V$. Note also that if two models do not omit a common variable, their prediction errors will be independent.

\footnotetext{
${ }^{17}$ We relax this assumption when we consider models of varying accuracy.
} 


\section{Linking Equilibrium Diversity to Collective Accuracy}

We now connect the equilibrium level of model diversity to collective accuracy. We first consider the case in which each predictive model has the same error variance, $V$, and the errors are independent, so that any two distinct models have zero error covariance. These two assumptions will hold provided that any two types consider the same number of attributes and between them consider all attributes. This assumption will be satisfied if each type considers all but one attribute.

Given the assumptions of common error variance and independence, the collective accuracy of the population depends on the error variance of the models, the size of the population, and the distribution across the various types. Even though each predictive model type is assumed to be error independent, conformity introduces non zero error covariance into the predictions because two individuals can use the same predictive model, i.e. be the same type.

We now state a claim that relates collective diversity, as measured by $\Delta$, model accuracy, as measured by $V$, and expected collective forecast error variance.

Claim 6 If each pair of predictive models has independent errors, then the expected squared collective error equals the ratio of individual accuracy, $V$, and collective diversity, $\Delta$. Formally:

$$
E[S q E(c)]=\frac{V}{\Delta}
$$

pf. Let $\varphi$ denote the probability that two individuals sampled randomly without replacement have the same model. We can write the general form of the average error covariance of the population as follows:

$$
\bar{C}=\frac{1}{N(N-1)} \sum_{i} \sum_{j, j \neq i}\left[\varphi E\left(e_{i}\right)^{2}+(1-\varphi) E\left(e_{i} e_{j}\right)\right]
$$

Note that if two individuals $i$ and $j$ have the same model, their error covariance equals the error variance of each individual, which by assumption equals $V$. Further, if two individuals have different models then given the error independence assumption, their error covariance equals 0.

$$
\bar{C}=\frac{\varphi}{N(N-1)} \sum_{i=1}^{N} \sum_{j, j \neq i} E\left(e_{i}\right)^{2}=\varphi V .
$$


The expected collective squared error can be written as follows:

$$
\begin{aligned}
E[S q E(c)] & =E\left[\frac{1}{N} \sum_{j=1}^{N} \chi_{j}(x)-\chi(x)\right]^{2}=\frac{1}{N^{2}} E\left[\sum_{j=1}^{N} e_{j}(x)\right]^{2} \\
& =\frac{1}{N} V+\frac{N-1}{N} \bar{C}=\left[\frac{1}{N}+\frac{N-1}{N} \varphi\right] V
\end{aligned}
$$

Note that $\frac{1}{\Delta}=\frac{1}{N}+\frac{N-1}{N} \varphi$ where $\frac{1}{\Delta}$ is the probability that two individuals sampled randomly with replacement have the same model, completing the proof.

We next extend this result to handle non error independent models. To compute the collective error, we now follow the same reasoning as the proof in the case of error independent models. This result extends the familiar bias variance decomposition result to distributions across models.

Claim 7 Assume that any two predictive models have the same error covariance, denoted by $C$, the expected collective error is given by the following expression:

$$
E[S q E(c)]=\frac{V}{\Delta}+\left(1-\frac{1}{\Delta}\right) C=\frac{1}{\Delta}(V-C)+C
$$

pf. As in the case of independent errors, if two individuals $i$ and $j$ use the same model, their error covariance also equals $V$. As before, this occurs with probability $\varphi=\frac{N}{\Delta(N-1)}-\frac{1}{(N-1)}$, which equals the probability that two individuals chosen without replacement have the same model. Now though, if two individuals have different models then their error covariance equals $C<V$. This occurs with probability $(1-\varphi)$. Therefore, the average expected error covariance equals

$$
\bar{C}=\frac{1}{N(N-1)} \sum_{i} \sum_{j, j \neq i}\left[\varphi V+(1-\varphi) C_{i j}\right]
$$

This expression can be rewritten as

$$
\bar{C}=\varphi V+(1-\varphi) C
$$


We can then compute the expected collective squared error as follows:

$$
E[S q E(c)]=\frac{1}{N} V+\frac{N-1}{N}(\varphi V+(1-\varphi) C)
$$

Rearranging terms gives the result.

To interpret this claim, consider how $C$ and $V$ depend on the number of common and idiosyncratic attributes. If there exist $B$ common attributes and $S$ idiosyncratic attributes then, $V=M-S-B$ and $C=M-2 S-B$. Note that this implies that giving each individual one more idiosyncratic attribute, i.e. making each individual more accurate in a unique way, has a substantial larger effect than if we gave each individual one more common attribute.

Claim ?? implies that collective accuracy is inversely related to the diversity index since $C<V$.

\section{Social Structure and Collective Accuracy}

In the previous two sections, we first derived explicit calculations for $\frac{1}{\Delta}$ as a function of the social structure, the population size, the number of models, the generating distribution, and the relative probability of experimentation and then derived a closed form solution for the expected squared error

of the population in terms of $\frac{1}{\Delta}$, the diversity index. In this section, we connect these two sets of results and show how collective accuracy depends on the attributes of the social process. We do so in the form of several corollaries. The first two corollaries are straightforward given our results from the previous two sections. Recall our notation that $N$ denotes the size of the population, $\nu$ denotes the rate of experimentation, and $K$ denotes the number of available predictive models. The first corollary states that breaking the population into isolated groups increases collective accuracy.

Corollary 1 For $N>1, \nu \in(0,1)$, and $K>1$, splitting a population into isolated non interacting groups reduces the expected squared error in the population's prediction.

The second corollary states that creating a hierarchy reduces the collective accuracy.

Corollary 2 For $N>1, \nu \in(0,1)$, and $K>1$, creating a hierarchical structure increases the expected 
squared error in the population's prediction.

The next three corollaries describe comparative statics. The proofs are in the appendix. The first of these three states that increasing the probability of experimentation increases expected accuracy but at a decreasing rate.

Corollary 3 For $N>1, \nu \in(0,1)$, and $K>1$, the expected squared error in the population's prediction decreases but at a decreasing rate in the probability of experimentation for all three social structures.

The next corollary states that making the generating process more diverse increases collective accuracy.

Corollary 4 For $N>1, \nu \in(0,1)$, and $K>1$, the expected squared error in the population's prediction decreases but at a decreasing rate in the diversity of the generating process for new models for all three social structures.

The final corollary states that increasing the population size increases accuracy.

Corollary 5 For $N>1, \nu \in(0,1)$, and $K>1$, the expected squared error in the population's prediction decreases but at a decreasing rate in the size of the population for all three social structures.

None of these corollaries runs counter to intuition. Creating distinct groups, increasing experimentation, increasing model diversity, and having a larger population all increase population diversity and therefore collective accuracy. And, creating a set of elites that everyone copies reduces diversity and with it accuracy. What's less clear from the comparative static analysis is the magnitude of these effects. How much does each effect collective accuracy? We consider that next.

\section{Comparative Statics}

To examine the effects of changing the parameters and the social structure on the equilibrium level of diversity and collective accuracy, we prove mathematical results and also perform numerical calculations. 
Both help to reveal the magnitude of effects. We restrict attention to error independent predictive models. 18

We divide our analysis into two parts. First, we consider large populations and show that model diversity swamps all other effects. We then consider smaller and more moderately sized populations and compare the relative effects of changing the social structure, the amount of model diversity, and the relative probabilities of experimentation on collective accuracy.

\section{Large Populations}

For large populations, the collective error depends on $D(G)$, the diversity of available predictive models of the generating process, and $V$ the variance of each predictive model. In other words, social structure and changing the probability of experimentation do not influence collective accuracy.

Claim 8 As $N \rightarrow \infty$ the expected squared error equals $\frac{V}{D(G)}$ for all three social structures.

pf. We first consider random mixing. From Claim ?? before, we have

$$
\frac{1}{\Delta}=1-\left[1-\frac{1}{N\left(2 \nu-\nu^{2}\right)+(1-\nu)^{2}}\right](1-f(G))
$$

Letting $N$ go to infinity, this reduces to

$$
\lim _{N \rightarrow \infty} \frac{1}{\Delta}=f(G)=\frac{1}{D(G)}
$$

Next, we consider breaking the population into $H$ groups. From Claim ?? before, we have

$$
\frac{1}{\Delta_{I}}=1-\left(1-\frac{1}{N\left(2 \nu-\nu^{2}\right)+H(1-\nu)^{2}}\right)(1-f(G))
$$

Letting $N$ go to infinity gives

$$
\lim _{N \rightarrow \infty} \frac{1}{\Delta_{I}}=f(G)=\frac{1}{D(G)}
$$

\footnotetext{
${ }^{18}$ The extension to the more general case is straightforward.
} 
Finally, if we assume that a fixed proportion of the population, $\alpha$, comprises the upper group, then from Claim ??, we have:

$$
\frac{1}{\Delta_{H}}=1-\left(1-\frac{1}{N}\right)\left(1-\frac{(1-\nu)^{2}}{\alpha N\left(2 \nu-\nu^{2}\right)+(1-\nu)^{2}}\right)(1-f(G))
$$

which as $N$ goes to infinity gives.

$$
\lim _{N \rightarrow \infty} \frac{1}{\Delta_{H}}=f(G)=\frac{1}{D(G)}
$$

By Claim ??, for all three social structures,

$$
\lim _{N \rightarrow \infty} E[S q E(c)]=\frac{V}{D(G)}
$$

Consider a population of 100,000 individuals, fifty models where models have an equal probability of being selected in the generating process, ${ }^{19}$ and a probability of experimentation of ten percent. If we normalize the variance to one $(V=1)$, dividing the population into 100 groups decreases the expected squared error by 0.0000002 and increasing the probability of experimentation to twenty percent decreases the expected squared error for the randomly mixed group by 0.00003 . By contrast, increasing the number of models $(K)$ from ten to fifty produces a decrease of 0.08 .

\section{Moderately Sized Populations $(N=1000)$}

We now consider moderately sized populations of one thousand individuals $(N=1000)$. These could correspond to all the employees in a firm or non profit organization, the active members of a political party, or the members of a small community.

In Figure ??, we plot collective accuracy as a function of the probability of experimentation for the three social structures assuming fifty models, fifty isolated groups, and one hundred elite individuals in the upper group. Notice first that breaking the population into isolated groups, in this case fifty, has very little effect on accuracy other than for low probabilities of experimentation.

Even though isolating groups increases diversity, it does so by very little. This result is counter-

\footnotetext{
${ }^{19}$ For all our numerical simulations, we make this assumption, i.e. $f(G)=\frac{1}{K}$.
} 
intuitive. It turns out that the smaller subpopulations have less capacity to maintain diversity and that this counteracts the diversity gain from isolation.

The logic is best seen in an example. Suppose that there exist eighty available models. A population of one thousand individuals may be able to maintain fifty types. A group of two hundred may only be able to maintain fifteen types. Five groups of size two hundred therefore might collectively only maintain fifty types: the same number as if the population were not subdivided. Thus, the diversity gain from isolation is offset by the loss in capacity to maintain diversity in the smaller subpopulations.

Note also that the hierarchical social structure produces a much less accurate population than the other two social structures. This finding agrees with our previous claims. What's surprising is the magnitude of the effect. The hierarchical social structure performs much worse. It would need a thirty five percent probability of experimentation to produce the accuracy the other social structures with five percent probabilities of experimentation.

That the effect of increasing the probability of experimentation is most pronounced for the hierarchical social structure, suggesting that in hierarchies, collective accuracy may depend critically on maintaining substantial levels of experimentation. This finding may partly explain why organizations encourage diversity and innovative thinking. Without such encouragement, diversity and collective accuracy plummet.

In contrast, the effect of experimentation on random groups exists only for very low probabilities of experimentation and there appears to be almost no effect when the population is broken into fifty isolated groups. This last result occurs because each of the fifty groups has only twenty members. Even with high levels of experimentation, groups of such small size support very little diversity. Put differently, the diversity that exists in the isolated social structure depends primarily on the differences between distinct groups and not on experimentation within the groups.

We next consider the effect of varying the number of isolated subpopulations. We do so to check if the lack of benefit from isolation was an artifact of choosing twenty subgroups. We find that not to be the case. For moderate sized populations, creating isolated subpopulations has almost no effect on collective accuracy as can be seen in Figure ?? which shows collective accuracy as the number of isolated groups increases from one to twenty five.

In Figure ??, we increase the number of available models from one to fifty. Here, as in the large population case, we see a substantial effect for all social structures. Thus, the number of available models 
appears to be extremely important for collective diversity. Note that the hierarchical structure would need fifty available models to be approximately as accurate as the other structures with only fifteen or twenty available models.

And, in Figure ??, we increase the size of the group of elites. As expected, making the group of elites larger reduces collective error. Less expected, the extent of the effect proves substantial. The magnitude of the effect becomes relatively larger when the group of elites is increased from very small (say around ten) to more moderately sized (around one hundred).

\section{Small Populations $(N=20)$}

Last, we consider populations of size twenty. A group of this size might be a management team, a board of directors, or a group of physicians. We find benefits from increasing the probability of experimentation, increasing the number of available models, and creating isolated subgroups. For example, Figure ?? shows the effect of increasing the probability of experimentation for populations of size twenty, ten possible models, four isolated groups, and an elite group of five individuals. The relatively large benefit for increasing the probability of experimentation occurs because small groups of people are unable to maintain much diversity otherwise.

As before, hierarchical structures need much more experimentation to maintain diversity. A hierarchical model with a thirty-five percent experimentation probability will be approximately as accurate as a random structure with a fifteen percent experimentation probability or isolated groups with a five percent probability.

Next, in Figure ?? we show the benefit of increasing the number of possible models. The benefit is substantial until the number of model approaches the population size. Notably, no amount of increase in the number of models allows the hierarchical structure to reach the accuracy of the other structure once there exist at least three available models.

Finally, Figure ?? shows how the collective error decreases as more isolated groups are created. Here we see a relatively large effect. The reason that isolation works for smaller populations is that the initial population is incapable of maintaining much diversity. Suppose for example that a group of twenty can only maintain six models in equilibrium. Ignoring the probability that two people choose the same model, by isolating each individual, the population would support twenty models. 


\section{Variation in Model Accuracy and Selection}

We now extend the framework to allow for models of differing accuracy and selection effects. Barring other model attributes such as salience or ease of recall, equal accuracy implies no selection effects. But the causality need not run in the other direction. There can exist variation in accuracy but no selection. For selection to occur, individuals would have to be aware that some models are more accurate than others. This may or may not be the case, as individuals may not have sufficient information to discern the accuracy of models.

To include for variation, we adopt the following convention. We assume that each predictive model belongs to a group and that within a group all models have identical accuracy. To make this formal, assume that the models can be portioned into $W$ bins denoted by $B_{1}$ through $B_{W}$ and that all models in a given bin have identical accuracy, where $V_{B_{w}}$ denotes the accuracy of bin $w$. To avoid overcomplicating the analysis, we assume that all models - even those in different groups - have independent errors. The extension to correlated errors is straightforward. We further assume that the model generating process $G$ follows a uniform distribution over the set of available models.

If we preclude selection effects but allow variation in model accuracy then our previous results extend. Let $\rho_{w}$ denote the proportion of available models in bin $B_{w}$ so that the average accuracy of a random collection of models equals

$$
\bar{V}_{B}=\sum_{w=1}^{W} \rho_{w} V_{B_{w}}
$$

We then have the following extension of Claim ??.

Claim 9 If there exist $W$ bins of models with accuracies $V_{B_{w}}$ for $w=1$ to $W$, if all models have pairwise independent errors, and if there exists no selection, then the expected squared collective error equals

$$
E\left[S q E_{B W}(c)\right]=\frac{\bar{V}_{B}}{\Delta}
$$

where $\Delta$ is the equilibrium diversity index of the population resulting from drifting as before. 
The intuition of Claim ?? is straightforward. Collective accuracy in equilibrium depends on average model accuracy and diversity of models in the population in equilibrium. Since there is no selection effect, the average model accuracy of the population should stay the same as that of all available models. The formal proof is in the appendix.

We can now add in selection effects. At one extreme, selection could be sufficiently strong that the only models that remain in the population belong to the most accurate bin. In that case, our previous analysis holds where the set of models consists only of those models in the most accurate bin and not the set of all models. In this case, selection drives out less accurate models, but drift determines how many of the most accurate models are in use.

The most interesting case allows for some selection. We capture this by assuming that individuals are more likely to copy more accurate models but that they experiment with models randomly. Let bins of models be ordered according to their accuracy so that $V_{B_{1}}<V_{B_{2}}<\ldots<V_{B_{W}}$. To avoid unnecessary complications, we also assume that each bin $B_{w}$ contains the same number of models so that each bin contains $\frac{1}{W}$ portion of the total available models.

Given $W$ bins and a relative selection effect for each bin it is a straightforward exercise to solve for the equilibrium proportion of models from that bin. Formally, let $\beta_{w}$ be a proxy for the fitness or selective pressure in the direction of models from bin $B_{w}$ such that $\beta_{1} \geq \beta_{2} \geq \ldots \geq \beta_{W}>0$. If $\rho_{w}$ denotes the equilibrium proportion of individuals in the population using models from bin $w,{ }^{20} \rho_{w}$ would have to satisfy the following equation:

$$
\rho_{w}=(1-\nu) \frac{\beta_{w} \rho_{w}}{\sum_{j=1}^{W} \beta_{j} \rho_{j}}+\frac{\nu}{W}
$$

The first term denotes the probability that a model from bin $B_{w}$ is copied by another individual. The second term equals the probability that a randomly selected model (an experiment) belongs to bin $B_{w}$. It can be shown that this equation has a unique positive solution. ${ }^{21}$ If there is no selection effect, that is, if all $\beta_{w}^{\prime}$ s are equal, then $\rho_{w}=\frac{1}{W}$ confirming our earlier intuition for Claim ??. Furthermore, when there is strong selection bias towards higher accuracy models so that $\beta_{w}$ is strongly decreasing in a decreasing rate in $w$, the equilibrium proportions will strongly skew to models from bins with higher

\footnotetext{
${ }^{20}$ A slight abuse of notation since we used the same symbol before for the portion of models in bin $w$ of all available models.

${ }^{21}$ As an example, if $W=2, \beta_{1}=2, \beta_{2}=1$ and $\nu=\frac{1}{3}$, then $\rho_{1}=0.7287$.
} 
accuracy.

To compute collective accuracy given selection, in addition to knowing the equilibrium proportion of population using models from each bin, we must also know the diversity of the population in equilibrium. We have the following:

Claim 10 Given selection based on $\beta=\left(\beta_{1}, \ldots, \beta_{W}\right)$, let $\Delta_{\beta}$ denote the diversity of the population in the equilibrium and $\bar{V}_{\beta}$ denote the average model accuracy of the population computed with equilibrium proportion $\rho_{w}$, the expected square error of the collective prediction equals

$$
E\left[S q E_{\beta}(c)\right]=\frac{\bar{V}_{\beta}}{\Delta_{\beta}}
$$

The proof for this claim is similar to that of Claim ??. It remains to derive the equilibrium level of diversity. As in the case without selection, we first solve for the equilibrium probability that two individuals have the same progenitor. That probability can be written in terms of $P_{\beta}^{s}$, the probability that two individuals copy the same individual, and $P_{\beta}^{d}$, the probability that two individuals copy different individuals in the presence of selection. With the equilibrium proportion of individuals from the population using models from bin $w$ being $\rho_{w}$, these probabilities can be computed as follows:

$$
P_{\beta}^{s}=\sum_{w=1}^{W}\left(\frac{\beta_{w} \rho_{w}}{\sum_{j=1}^{W} \beta_{j} \rho_{j}}\right)^{2}(1-\nu)^{2} \frac{1}{\rho_{w} N}
$$

and

$$
P_{\beta}^{d}=(1-\nu)^{2}-P_{\beta}^{\mathbf{s}}
$$

Claim 11 Let $a_{\beta}$ denote the stochastic equilibrium probability that two randomly drawn (without replacement) individuals from the population have the same progenitor. In equilibrium

$$
a_{\beta}=\frac{P_{\beta}^{\mathbf{s}}}{1-P_{\beta}^{d}}
$$

pf. The difference equation for the probability that two randomly drawn (without replacement) individuals have the same progenitor can be written as follows:

$$
a_{\beta}^{\prime}-a_{\beta}=-a_{\beta}\left[\nu^{2}+2 \nu(1-\nu)+P_{\beta}^{d}\left(1-a_{\beta}\right)\right]+\left(1-a_{\beta}\right)\left(P_{\beta}^{\mathbf{s}}+P_{\beta}^{d} a_{\beta}\right)
$$


This can be simplified to

$$
a_{\beta}^{\prime}-a_{\beta}=-a_{\beta}+\left(P_{\beta}^{\mathbf{s}}+P_{\beta}^{d} a_{\beta}\right)
$$

The equilibrium result follows.

We can complete the analysis by connecting $a_{\beta}$ to the equilibrium diversity $\Delta_{\beta}$. Using the same construction used in Claim ??, one can prove the following:

Claim 12 Given selection, the equilibrium probability that two randomly chosen (with replacement) individuals have the same type equals

$$
\frac{1}{\Delta_{\beta}}=1-\left(1-\frac{1}{N}\right)\left(1-a_{\beta}\right)(1-f(G))
$$

One implication of these claims is that selection proves to be a two edged sword. If the selection effects are strong and bin sizes are small, then $P_{\beta}^{\mathbf{s}}$, the probability that two individuals copy the same person will be high, which implies that $a_{\beta}$, the probability that two models have the same progenitor will also be high. This lack of diversity will reduce accuracy. Based on our previous analysis, as well as results from a similar model using a computational model (Hong, Page, and Riolo 2012) it appears that whether this decrease outweighs the gain from reliance on more accurate models depends on the strength of selection relative to the probability of experimentation. If selection is strong, unless the best bin includes many models, selection will make the population less accurate.

\section{Discussion}

In this paper, we have presented a framework that enables us to derive the equilibrium diversity of predictive models as a function of the probability of experimentation and conformity, social structure, the diversity of available models, and population size. We then extended the bias variance decomposition theorem to show how collective accuracy can be written as a function of the diversity index. Connecting this result to our first set of results allows us to express the expected collective accuracy of a population in terms of the aforementioned characteristics of the system.

That analysis allows us to show that for large populations the diversity and accuracy of available models determines collective accuracy. Social structure hardly matters at all, nor does changing the probability of experimentation. For moderately sized populations, raising the probability of experi- 
mentation begins to play a role particularly for hierarchical social structures. This finding provides an insight into why moderately sized organizations place such an emphasis on innovative thinking increasing experimentation produces more diversity and better predictions.

Surprisingly, we find that creating distinct non interacting populations (fire walling) does not increase diversity except in the case of very small populations (around twenty people). Even in that case, fire walling is not a particularly effective strategy. Having more models and increasing experimentation are stronger levers.

Our finding that the number of available models has such a large effect in all cases suggests a social benefit from the production of alternative models. This diversity of models could arise naturally - from different information or from people transferring models from other domains. But model diversity could also be encouraged through incentives to think differently.

In addition to the first order effects just described, we also derived second order effects. We find that efforts to increase collective accuracy, be it by increasing model diversity, population size, or social structure, do so with diminishing returns. These second order results suggest that after increasing the number of models initially, it makes sense to increase rates of experimentation, and to change the social structure.

These findings were all derived assuming equally accurate models and no selection. Adding heterogeneity without selection does not have any effect on the results in expectation other than increasing variation. When we add selection as well, we find that it can lower collective accuracy by reducing diversity.

These preliminary findings suggest the value of a more general analysis of the optimal balance between selection and drift. The current model might also be extended in at least four other ways. One could analyze more detailed network structures. One could consider alternative formalisms of the types of models that people employ and how those aggregate. We have used an interpreted signal model but one could equally well use generated signals (Hong and Page 2009). One could also link the social process model to geographic locations. Finally, one could reformulate the hierarchical model and assume that the elites draw their models from a different and more accurate collation of models. This assumption could be reasonable if elites gain their status based on ability. In that case, the models copied from the elites would be more accurate than those the non elites construct on their own. This would create a bias in favor of less experimentation at the lower level. Our guess is that one result of that model would be 
that elites should experiment more than non elites. ${ }^{22}$

Though in this paper we only consider the effects of diversity on the ability of a collective to perform well on predictive tasks, population level cognitive diversity has much broader organizational and societal implications. Here, we have focused on prediction but one could also consider auction markets, problem solving (Page 2007) or responsiveness to shocks.

For example, a central research area within organizational behavior considers how cognitive diversity influences team performance (Jehn, Northcraft, and Neale 1999). ${ }^{23}$ Just as diverse predictive models improve collective prediction, diverse perspectives, heuristics, and mental models improve collective problem solving and the ability of organizations to innovate (Hong and Page 2001, Page 2007, Nooteboom et al 2007). Furthermore, network structure has been linked to the diversity of information and knowledge that managers receive and in turn tied to managerial performance (Rodan and Galunic 2004). For a manager to tap into diverse ways of thinking, the organization must be capable of producing and maintaining diversity. That level diversity - the different ways of thinking held by the population emerges from a social process.

In light of the many implications of population level diversity, the most important methodological contribution of the paper may be that it demonstrates the possibility of endogenizing distributions across types. As mentioned in the introduction, nearly all papers that address information aggregation, whether they be in economics, finance, political science, or psychology, take the distribution of signals as fixed (Feddersen and Pesendorfer 1997, Golub and Jackson 2010). Often, types correspond to information, beliefs, or ways of thinking. In so far as results of models depend on the type distribution, we should seek to understand how the distributions come to be. The framework that we presented in this paper provides one approach to connecting distributions of types to underlying social processes and social structure.

\section{References}

[1] Armstrong, J. Scott. (2001). Combining forecasts. Principles of Forecasting: A Handbook for Researchers and Practitioners. Kluwer Academic Publishers, Norwell, MA.

\footnotetext{
${ }^{22}$ We thank a referee for this excellent idea.

${ }^{23} \mathrm{~A}$ number of papers consider cognitive diversity together with identity diversity. See, for example, Mannix and Neale (2005) and De Wit, et al. (2012). Our techniques would only apply to acquired and transmittable differences.
} 
[2] Ashton, R.H. (1986). "Combining the judgments of experts: How many and which ones?" Organizational Behavior and Human Decision Processes 38(3) 405-414.

[3] Batchelor, Roy, Pami Dua. (1995). "Forecaster diversity and the benefits of combining forecasts." Management Science 41(1) 68- 75.

[4] Bednar, Jenna, Aaron Bramson, Andrea Jones-Rooy, and Scott E. Page. (2010). "Emergent Cultural Signatures and Persistent Diversity: A Model of Conformity and Consistency." Rationality and Society 22(4):407-44.

[5] Bentley, R.A., Hahn, M.W., and Shennan, S.J. (2004). "Random drift and culture change." Proceedings of the Royal Society B, 271, 1443-1450.

[6] Bentley, R.A. P. Ormerod, and M. Batty (2011). "Evolving social influence in large populations," Behavioral Ecology and Sociobiology, 65:537-546

[7] Breiman, L., (1996). "Bagging Predictors", Machine Learning , 24(2), pp.123-140.

[8] Cavalli-Sforza, L.L., and Feldman, M.W. (1981). Cultural Transmission and Evolution: A Quantitative Approach. Princeton University Press, Princeton, NJ.

[9] De Wit, F.R.C. L L. Greer, and Karen A. Jehn. (2012). "A meta-analysis of the relationships between diversity, conflict, and team performance." Journal of Applied Psychology 92.2: 360-390.

[10] Economo, E., and Keitt, T.H. (2008). "Species diversity in neutral metacommunities: a network approach." Ecology Letters 11 (1): 52-62.

[11] Feddersen T, and Pesendorfer W. (1997). "Voting Behavior and Information Aggregation in Elections with Private Information." Econometrica 65 (5): 1029 -1058.

[12] Friedman, D. (1998). "On economic applications of evolutionary game theory". Journal of Evolutionary Economics. 8: 15-43.

[13] Golub B, and Jackson M. (2010). "Naive Learning in Social Networks: Convergence, Influence and the Wisdom of Crowds. "American Economic Journal: Microeconomics 2(1): 112-149.

[14] Grofman, B., Owen, G, and Feld, S. (1983). "Thirteen theorems in search of the truth" Theory and Decision 15:3 pp 261-278. 
[15] Hartl, D.L., and Clark, A.G. (1997). Principles of Population Genetics. Sinauer Associates. Sunderland, MA.

[16] Hong, L, and Page, S. (2001). "Problem Solving by Heterogeneous Agents." Journal of Economic Theory 97: 123-163.

[17] Hong, L, and Page, S. (2009). "Interpreted and Generated Signals." Journal of Economic Theory 144: $2174-2196$.

[18] Hong, L, Page, S. and Riolo, M. (2012). "Incentives, Information, and Emergence Collective Accuracy." Managerial and Decision Economics 33: 323-334.

[19] Hubbell, S.P. (2001). The Unified Neutral Theory of Biodiversity and Biogeography. Princeton University Press. Princeton, NJ.

[20] Huckfeldt, R. Robert, Paul E. Johnson, John D. Sprague (2004). Political Disagreement: The Survival of Diverse Opinions Within Communication Networks Cambridge University Press, Cambridge, England.

[21] Jehn, Karen A., Northcraft, Gregory B., and Neale, Margaret A. (1999). "Why differences make a difference: A field study of diversity, conflict, and performance in workgroups." Administrative Science Quarterly, 44, 741-763.

[22] Kimura, M. (1984). The Neutral Theory of Molecular Evolution. Cambridge University Press. Cambridge, England.

[23] Kimura, M., and Crow, J.F. (1964). "The Number of Alleles That Can be Maintained in a Finite Population" Genetics 49 (4): 725-738.

[24] Lamberson, P.J. and Page, S. (2012). "Optimal Forecasting Groups" Management Science 58: 791804.

[25] Le Bon, G. (1895) The Crowd: A Study of the Popular Mind public domain.

[26] Mannix, E. A.,and Neale, M. A. (2005). "What differences make a difference? The promise and reality of diverse teams in organizations". Psychological Science in the Public Interest, 6, 31-55.

[27] Maynard Smith, John (1982). Evolution and the Theory of Games, Cambridge University Press. 
[28] Nooteboom, Bart, Wim Van Haverbeke, Geert Duysters, Victor Gilsing, Ad van den Oord. (2007). "Optimal cognitive distance and absorptive capacity." Research Policy 36(7) 1016?1034.

[29] Ober, J. (2008). Democracy and Knowledge: Innovation and Learning in Classical Athens. Princeton University Press, Princeton.

[30] Ormerod, P. (2012). Positive Linking: How Networks Can Revolutionise the World Faber and Faber, London.

[31] Page, S. (2008). The Difference: How the Power of Diversity Creates Better Groups, Firms, Schools, and Societies, Princeton University Press.

[32] Page, Scott E (2010) Diversity and Complexity Princeton University Press, Princeton, NJ.

[33] Page, Scott E (2014) "Where diversity comes from and why it matters?" European Journal of Social Psychology 44 (4) 267-279.

[34] Piketty, T. (1999) "The information-aggregation approach to political institutions" European Economic Review 43: 7910̃ 800

[35] Reinhart, C. and K. Rogoff (2009). This Time is Different: Eight Centuries of Financial Folly Princeton University Press. Princeton, NJ.

[36] Rodan, Simon and C. Galunic, (2004). "More than network structure: how knowledge heterogeneity influences managerial performance and innovativeness" Strategic Management Journal25: 6, pp $541 ? 562$.

[37] Von Hayek, F. (1945). "The Use of Knowledge in Society." American Economic Review, 4 pp 519530.

[38] Welinder P., S. Branson, S. Belongie, P. Perona, (2010). "The Multidimensional Wisdom of Crowds." Conference on Neural Information Processing Systems (NIPS).

[39] Whitlock, M., and Barton, N.H. (1997). "The Effective Size of a Subdivided Population" Genetics 146 (1): 427-441.

[40] Whyte, G. (1989). "Groupthink reconsidered." The Academy of Management Review, Vol. 14(1): 4056. 
[41] Young, H.P. (2009). "Innovation Diffusion in Heterogeneous Populations: Contagion, Social Influence, and Social Learning." American Economic Review, 99, 1899-1924.

[42] Young, H.P. (2011). "The Dynamics of Social Innovation" Proceedings of the National Academy of Sciences108, No. 4, 21285-21291.

\section{Appendix}

In this appendix, we prove Corollaries 3, 4 and 5. Since $E[S q E(c)]=\frac{V}{\Delta}+\left(1-\frac{1}{\Delta}\right) C$ and $C<V$, we only need to show that for each of the three cases (random, isolated groups, hierarchy), the first derivative of

$\frac{1}{\Delta}$ is negative and the second derivative is positive with respect to each of the parameters $\nu, D(G)$, and $N$.

Corollary 3 For $N>1, \nu \in(0,1)$, and $K>1$, the expected squared error in the population's prediction decreases but at a decreasing rate in the probability of experimentation for all three social structures.

pf. we can compute the first and second derivatives w.r.t $\nu$ for each of the three social structures as follows:

Random: The first derivative is given by

$$
-\frac{2(1-f(G))(N-1)(1-\nu)}{\left[N-(N-1)(1-\nu)^{2}\right]^{2}}<0
$$

and the second derivative by

$$
\frac{2(1-f(G))(N-1)\left[N+3(N-1)(1-\nu)^{2}\right]}{\left[N-(N-1)(1-\nu)^{2}\right]^{3}}>0
$$

Isolated Groups: The first derivative is given by

$$
-\frac{2(1-f(G))(N-H)(1-\nu)}{\left[N-(N-H)(1-\nu)^{2}\right]^{2}}<0
$$

and the second derivative by 


$$
\frac{2(1-f(G))(N-H)\left[N+3(N-H)(1-\nu)^{2}\right]}{\left[N-(N-H)(1-\nu)^{2}\right]^{3}}>0
$$

Hierarchy: The first derivative is given by

$$
-\frac{2(1-f(G))\left(1-\frac{1}{N}\right) U(1-\nu)}{\left[U-(U-1)(1-\nu)^{2}\right]^{2}}<0
$$

and the second derivative by

$$
\frac{2(1-f(G))\left(1-\frac{1}{N}\right) U\left[U+3(U-1)(1-\nu)^{2}\right]}{\left[U-(U-1)(1-\nu)^{2}\right]^{3}}>0
$$

Therefore Corollary 3 holds.

Corollary 4 For $N>1, \nu \in(0,1)$, and $K>1$, the expected squared error in the population's prediction decreases but at a decreasing rate in the diversity of the generating process for all three social structures. pf: Recall the expression for $\frac{1}{\Delta}$ in the random case.

$$
\frac{1}{\Delta}=1-\left[1-\frac{1}{N\left(2 \nu-\nu^{2}\right)+(1-\nu)^{2}}\right]\left(1-\frac{1}{D(G)}\right)
$$

It follows that the first and second derivatives of $\frac{1}{\Delta}$ w.r.t. $D(G)$ have the same sign properties as the derivatives of $\frac{1}{D(G)}$. The result follows by inspection. Similar proofs hold for the other two social structures.

Corollary 5 For $N>1, \nu \in(0,1)$, and $K>1$, the expected squared error in the population's prediction decreases but at a decreasing rate in the size of the population for all three social structures.

pf. Note that the first and second derivatives for $\frac{1}{\Delta}$ w.r.t. $N$ for all three cases have the same sign properties as the derivatives of $\frac{1}{a N+b}$ where $a$ and $b$ are constants, $a>0$ and $b \geq 0$. The result then follows by inspection. 
Proof of Claim ??: Recall that $\varphi$ denotes the probability that two individuals sampled randomly without replacement have the same model in equilibrium. By assumption, if two individuals $i$ and $j$ have the same model, their error covariance equals the error variance of each which will be $V_{B_{w}}$ if the model belongs tobin $B_{w}$. Further, if two individuals have different models then given error independence, their error covariance equals 0 . As each model is equally likely to have a given concentration in the population, conditional on two people having the same model, the probability that the model is in bin $B_{w}$ equals $\rho_{w}$. We can then write the expected error covariance of the population as follows:

$$
\overline{C_{B W}}=\frac{\varphi}{N(N-1)} \sum_{i=1}^{N} \sum_{j, j \neq i} E\left(e_{i}\right)^{2}=\varphi \sum_{w=1}^{W} \rho_{w} V_{B_{w}}=\varphi \bar{V}_{B}
$$

The expected collective squared error can then be written as follows:

$$
\begin{aligned}
E\left[S q E_{B W}(c)\right] & =E\left[\frac{1}{N} \sum_{j=1}^{N} \chi_{j}(x)-\chi(x)\right]^{2}=\frac{1}{N^{2}} E\left[\sum_{j=1}^{N} e_{j}(x)\right]^{2} \\
& =\frac{1}{N}\left(\bar{V}_{B}\right)+\frac{N-1}{N} \overline{C_{B W}}=\left[\frac{1}{N}+\frac{N-1}{N} \varphi\right] \bar{V}_{B}
\end{aligned}
$$

Note that $\frac{1}{\Delta}=\frac{1}{N}+\frac{N-1}{N} \varphi$ where $\frac{1}{\Delta}$ is the probability that two individuals sampled randomly with replacement have the same model. This completes the proof. 

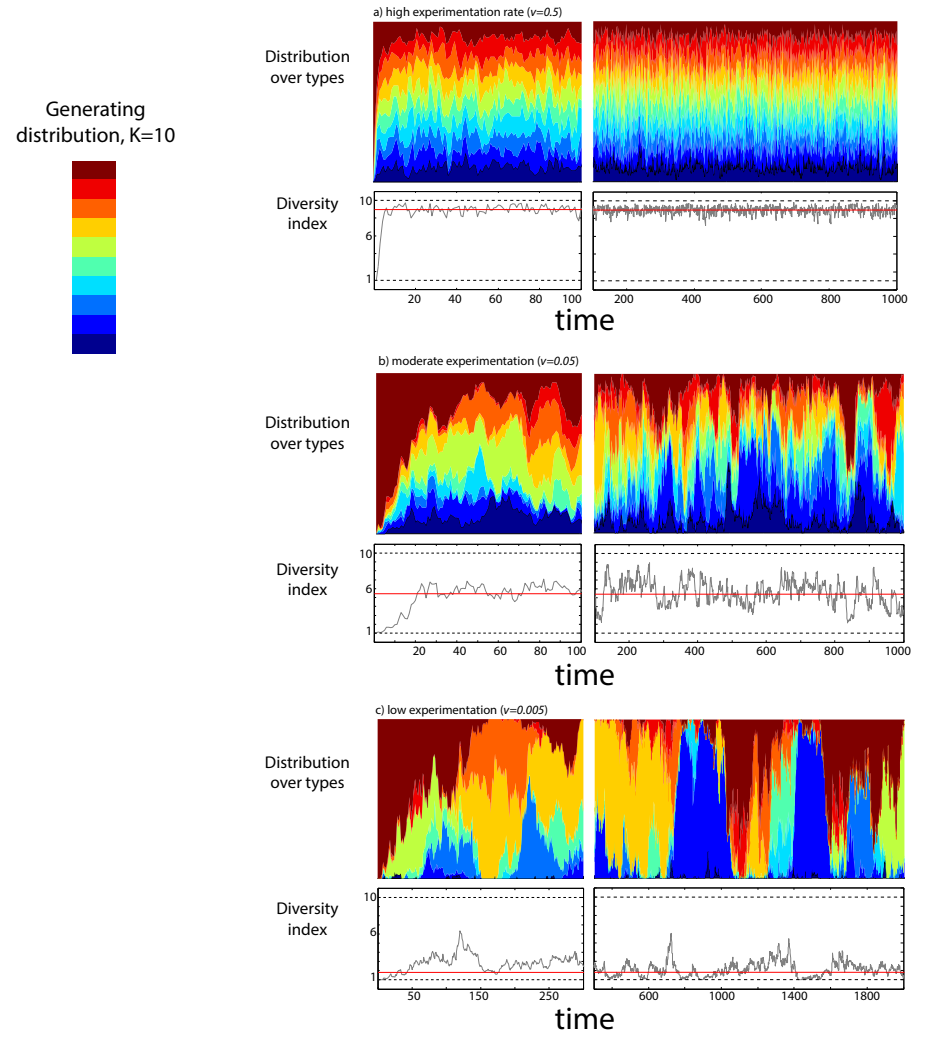

Figure 2: Sample Dynamic Paths For Various Levels of Experimentation 


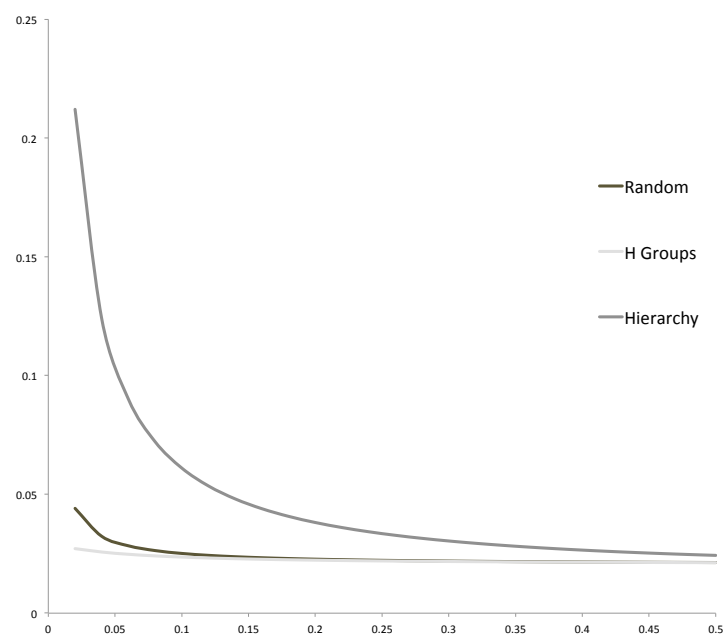

Figure 3: Changing the probability of experimentation: $V=1, N=1000, K=50, H=50, U=100$ Moderate Sized Groups

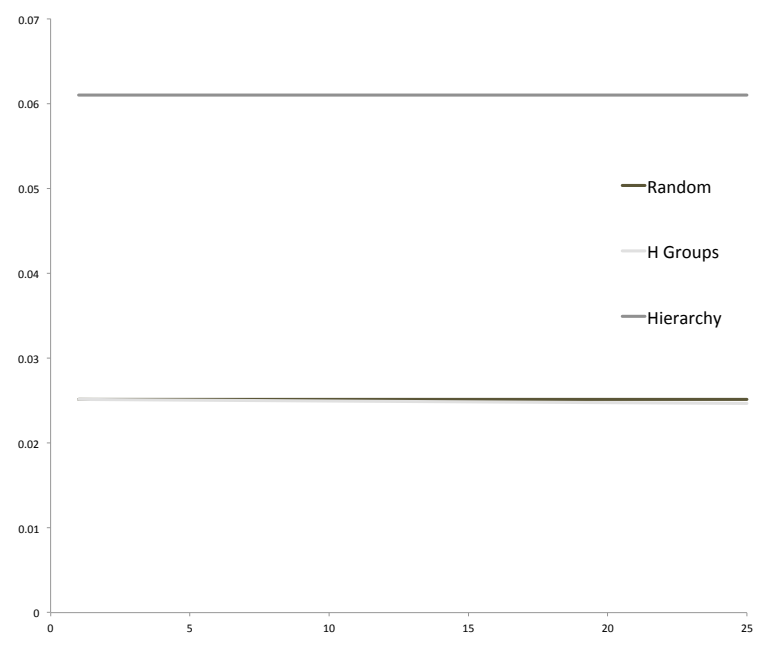

Figure 4: Increasing the Number of Isolated Groups $V=1, N=1000, K=50, U=100, \nu=0.1$ Moderate Sized Population 


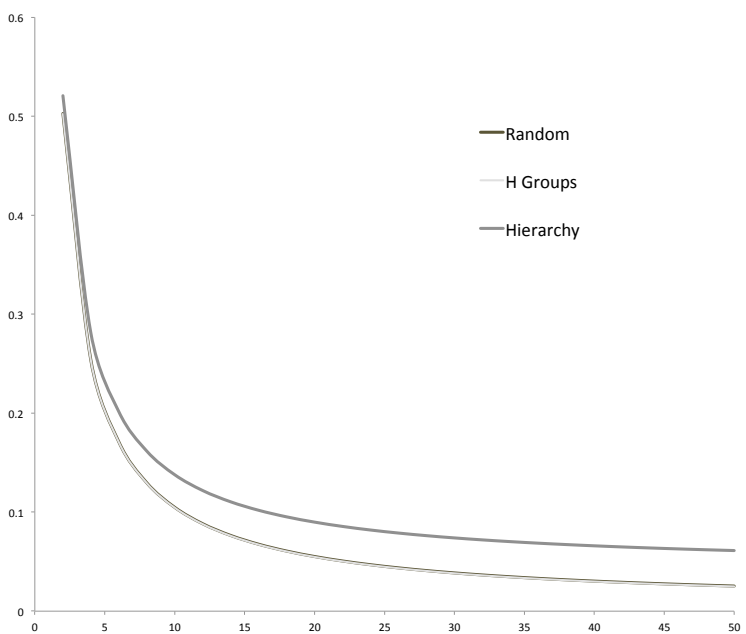

Figure 5: Increasing the Number of Models (K) $V=1, N=1000, H=20, U=100, \nu=0.1$ Moderate Sized Population

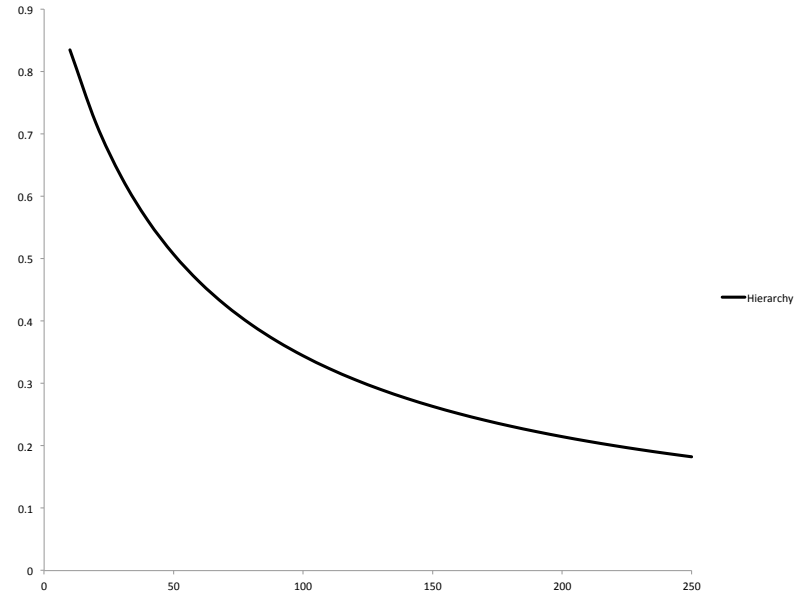

Figure 6: Increasing the Number of Elites (U) $V=1,: N=1000,: K=50, \nu=0.1$ Moderate Sized Population 


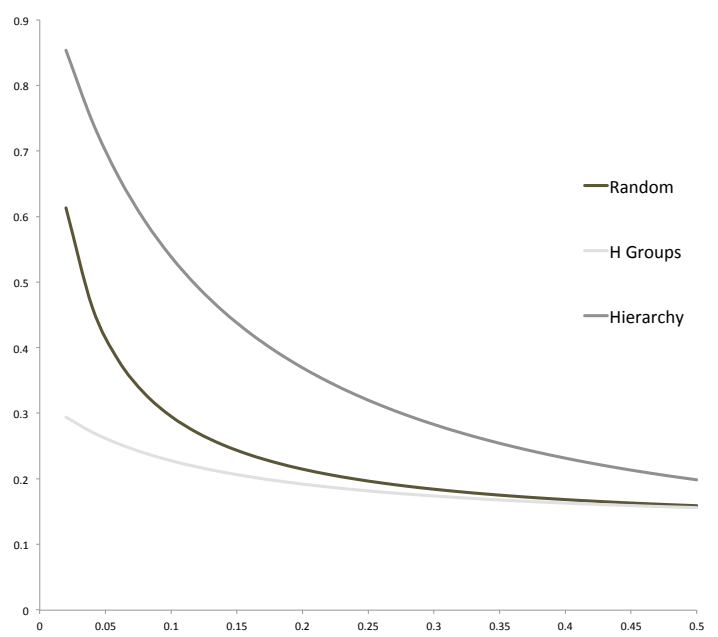

Figure 7: Increasing the probability of experimentation $(\nu) V=1,: N=20,: K=10,: H=4,: U=5$ Small Population

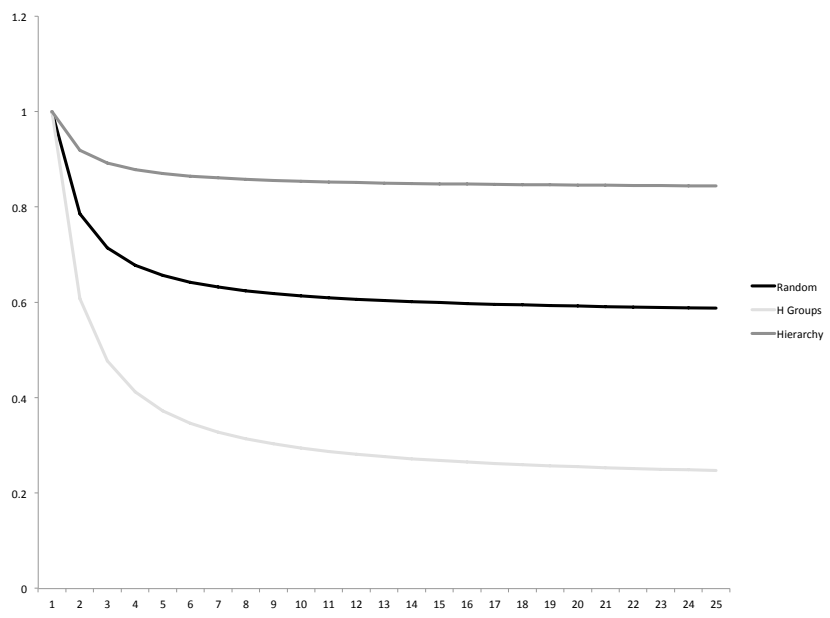

Figure 8: Increasing the number of models $(K): V=1, N=20, H=4, \nu=0.02, U=5$ Small Population 


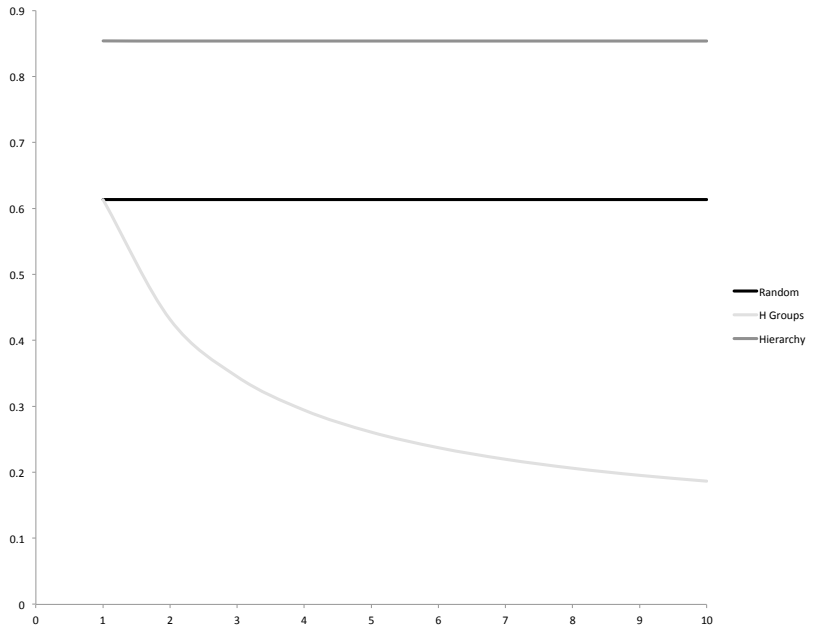

Figure 9: Increasing the number of isolated Groups $(H): V=1, N=20, K=10,, U=5, \nu=0.02$ Small Population 